\title{
Proposed revisions to the EU clinical trials directive-Comments from the European Resuscitation Council
}

\author{
Gavin D. Perkins*, Leo Bossaert, Jerry Nolan, Rudolph W. Koster, Koen Monsieurs, Maaret Castren, \\ On behalf of the European Resuscitation Council ${ }^{a}$
}

Drie Eikenstraat 661, 2650 Edegem, Belgium

\section{A R T I C L E I N F O}

Article history:

Received 21 January 2013

Accepted 21 January 2013

An editorial written by Fritz Stertz et al. in 2002 and published in this journal was one of the first to highlight concerns about the introduction of a new European Union (EU) clinical trials directive (Clinical Trials Directive 2001/20/EC) particularly for research in emergency settings. ${ }^{1}$ The directive sought "to simplify and harmonize the administrative provisions governing clinical trials in the European Community, by establishing a clear, transparent procedure for conducting studies involving investigational medicinal products". It is widely acknowledged that the Directive brought in unnecessary administrative and regulatory burdens. The inflexibility of the Directive led to individual countries creating local legislation in an attempt to facilitate emergency research, which increased variation between countries, and added delays and increased costs for researchers. ${ }^{2}$ A group of clinicians, ethicists and lawyers (the 'Vienna Initiative to save European Research' (VISEAR) investigators) produced a consensus document to promote a unified approach to local legislation, ${ }^{3}$ although the recommendations were never fully implemented.

The EU announced its intent to revise the Clinical Trials Directive 2001/20/EC in July 2012. ${ }^{4}$ The consultation paper draws specific attention to research in patients without capacity stating: "Regarding clinical trials in emergency situations, Directive 2001/20/EC does so far not address the specific situation where, because of the urgency of the situation, it is impossible to obtain free and informed consent from the subject or the legal representative ('clinical trials in emergency situations'). To address this, specific provisions on clinical trials in emergency situations have been added in line with existing international guidance documents on this issue."

\footnotetext{
* Corresponding author. Tel.: +44 07971811292.

E-mail address: g.d.perkins@warwick.ac.uk (G.D. Perkins).

a Appendix A (the list of the writing group members).
}

Article 32 addresses clinical trials in emergency settings. Paragraph 1 sets out that informed consent may be obtained after the start of the clinical trial provided that all of the following conditions are fulfilled:

(a) due to the urgency of the situation, caused by a sudden lifethreatening or other sudden serious medical condition, it is impossible to obtain prior informed consent from the subject and it is impossible to supply prior information to the subject;

(b) no legal representative is available;

(c) the subject has not previously expressed objections known to the investigator;

(d) the research relates directly to a medical condition which causes the impossibility to obtain prior informed consent and to supply prior information;

(e) the clinical trial poses a minimal risk to, and imposes a minimal burden on, the subject.

The ERC welcomes revisions to the EU Directive that are designed to facilitate research in emergencies whilst at the same time safeguarding patients. However we express concerns about the potential for misinterpretation of specific terminology. Specifically paragraph 1 , subsection (a), "due to the urgency of the situation, caused by a sudden life-threatening or other sudden serious medical condition". Subjects may become incapacitated either as a consequence of the underlying medical condition or its treatment. For example, a subject with severe asthma may require deep sedation to enable artificial ventilation to prevent cardiac arrest. In this case, it is the treatment (i.e. sedation), not the underlying condition that has rendered the subject incapacitated.

Sub-section (b), which demands that "no legal representative" is present before the emergency research provision can be applied, is problematic. First, even if a legal representative is present, there is often uncertainty if he would have sufficient mental capacity at 
the time of a relatives cardiac arrest to provide a considered view of the patients wishes. Second, in emergencies, where immediate treatment is time critical (e.g. in cardiac arrest), delays of even a few seconds in administering treatment substantially reduce the chances of survival. ${ }^{5,6}$ Therefore, delaying treatment to obtain consent would be harmful and, therefore, unethical.

Finally, subsection (d) requires that the research relates directly to a medical condition which makes it impossible to obtain prior informed consent. Medical conditions leading to critical illness and loss of capacity rarely occur in isolation. For example, if a subject develops acute renal failure as a consequence of cardiac arrest, the renal failure per se is not the cause of the incapacity but research into the treatment of renal failure is important. Under the above proposal there would be uncertainty about the legality of such research.

The European Resuscitation Council has written formally to the EU Standing Committee on Environment, Public Health and Food Safety to express their concerns. We also encourage individual researchers and national resuscitation councils to engage in dialogue with their local regulators to highlight the potential for misinterpretation and to seek clarification of wording before the Directive is finalized.

\section{Conflict of interest statement}

GDP, JPN, MC, KM, RWK LB are members of the Board of the European Resuscitation Council.

$\mathrm{MC}$ is a member of board of Falck Foundation.

Provenance and Peer Review: Commissioned, Not externally peer reviewed.

\section{Appendix A. Signatories from the ERC Executive Committee}

Janusz Andrès, Symposium Kraków, Poland; Luciano Anselmi, NRC, Switzerland; Jan Bahr, Director of Congresses, Germany; John Ballance, Representative of the ICC, UK; Michael Baubin, Congress
Vienna, Austria; Stefan Beckers, NRC,Germany; Dominique Biarent, ERC Honorary Secretary Belgium; Leo Bossaert, Representative of the Advisory Committee, Belgium; Antonio Caballero, NRC, Spain; Alain Cariou, ESICM, France; Pascal Cassan, European Red Cross National Societies, France; Maaret Castrén, ERC Chair, Sweden; Diana Cimpoesu, NRC, Romania; Kubilay Demirag, Turkey, Turkey; Manar Elkholy, vice-Chair ICC ILS, Egypt; Marios Georgiou, Director External Affairs, Cyprus; Tony Handley, Treasurer, UK; Tony Hosmans, NRC, Belgium; Silvija Hunyadi-Antičević, NRC Representative, Croatia; Uwe Kreimeier, Associate Representative of the Gold Members, Germany; Torsten Lauritsen, NRC, Denmark; Kristian Lexow, NRC, Norway; Freddy Lippert, Associate Representative of the Gold Members, Denmark; Carsten Lott, Chair of ICC ALS, Germany; Ian Maconochie, Representative of the WG, UK; Koenraad Monsieurs, Director of Guidelines and ILCOR, Belgium; Nikolaos Nikolaou, ESC, Greece; Jeremy Nolan, ERC Chief Editor, UK; Peter Paal, Associate Representative of the Gold Members, Austria; Gavin Perkins, Chair of WG BLS AED, UK; Thomas Rajka, NRC, Norway; Ferenc Sari, Associate Representative of the Gold Members, Sweden; Anna Spiteri, NRC, Malta; Hildigunnur Svavarsdóttir, Effective NRC representative, Iceland; Caroline Telion, NRC, France; Anatolij Truhlar, NRC, Czech Republic; Patrick Van De Voorde, Chair of ICC EPLS, Belgium; Henrik Wagner, NRC, Sweden.

\section{References}

1. Sterz F, Singer A, Bottiger B, et al. A serious threat to evidence based resuscitation within the European Union. Resuscitation 2002;53:237-8.

2. Lemaire F, Bion J, Blanco J, et al. The European Union Directive on Clinical Research: present status of implementation in EU member states' legislations with regard to the incompetent patient. Intensive Care Med 2005;31:476-9.

3. Liddell K, Chamberlain D, Menon DK, et al. The European Clinical Trials Directive revisited: the VISEAR recommendations. Resuscitation 2006;69:9-14.

4. Revision of the Clinical trials Directive: Adoption of the proposal for a "Clinical Trials Regulation". 2012. (Accessed at http://ec.europa.eu/health/human-use/ clinical-trials/index_en.htm - rlctd.).

5. Chan PS, Krumholz HM, Nichol G, Nallamothu BK. Delayed time to defibrillation after in-hospital cardiac arrest. N Engl J Med 2008;358:9-17.

6. Cheskes S, Schmicker RH, Christenson J, et al. Perishock pause: an independent predictor of survival from out-of-hospital shockable cardiac arrest. Circulation $2011 ; 124: 58-66$ 